\title{
Photochemical Oxidation of Poly(dimethylsiloxane) Surface and Subsequent Coating with Biomimetic Phosphorylcholine Polymer
}

\author{
Yoshiteru Hamada*, Takao Ono**, Takanori Akagi** ***, \\ Kazuhiko Ishihara $* * * * * *$ and Takanori Ichiki* $* * * * *$ \\ *Department of Materials Engineering, School of Engineering, \\ The University of Tokyo, 7-3-1 Hongo, Bunkyo-ku, 113-8656, Japan \\ **Department of Bioengineering, School of Engineering, \\ The University of Tokyo, 2-11-16 Yayoi, Bunkyo-ku, 113-8656, Japan \\ ***Center for NanoBio Integration, The University of Tokyo, \\ 7-3-1 Hongo, Bunkyo-ku, 113-8656, Japan
}

\begin{abstract}
As a material for fabricating biomicrosystem devices called lab-on-a-chip or micro total analysis system ( $\mu$ TAS), poly(dimethylsiloxane) (PDMS) has been widely used. Generally, the devices used in biological applications require some surface modification in order to control the hydrophilicity and nonspecific adsorption of biomolecules on their surfaces. However, it is difficult to achieve surface modification that remains sufficiently stable over long enough duration for use in PDMS microfluidic devices. In this paper, we report a simple but reliable method of applying a surface coating of biomimetic polymer to PDMS surfaces by the combinative use of photochemical surface oxidation by VUV irradiation and subsequent coating of phospholipid copolymer containing 2-methacryloyloxyethyl phosphorylcholine (MPC) and 3-methacryloxytriethoxysilane (METESi). The effectiveness of this coating process has been demonstrated by a significant reduction in the nonspecific adsorption of serum albumin and the formation of a micropatterned surface coating using a photomask.
\end{abstract}

Keywords: VUV irradiation, surface modification, poly(dimethylsiloxane), phosphorylcoline polymer

\section{Introduction}

Since the late 90 's, increasing attention has been directed towards the research and development of microfluidic systems for chemical and biological analyses, which are often called micro total analysis systems ( $\mu$ TASs) or lab-on-a-chip (LOC). In the prototyping research of these microfluidic devices, silicone elastomer, poly(dimethylsiloxane) (PDMS) (chemical formula: $-\mathrm{O}-\left[\left(\mathrm{CH}_{3}\right)_{2} \mathrm{Si}-\mathrm{O}\right]_{\mathrm{n}}-$ ), has been widely used owing to the ease of micropattern fabrication at low cost, resistance to various chemicals and $\mathrm{pH}$ environments, and excellent transparency in the UV-vis range [1, 2]. Its marked gas permeability and biocompatibility have, in particular, drawn attention to the integration of biological applications into PDMS devices [3, 4].
However, PDMS is rather hydrophilic in nature. Thus the problem of nonspecific adsorption of biomolecules on microchannel walls without any special surface treatment often arises. Owing to the very small sample volume and the supersensitivity of analysis using a microfluidic system, the adsorption of biomolecules on PDMS significantly affects the quantitative measurement.

In order to overcome this issue, various solutions have been proposed for tailoring the PDMS surface properties. Surface oxidation using oxygen plasma or UV curing is most often employed in rapid prototyping of microfluidic devices mainly because of its simplicity and compatibility with the sealing process of microfluidic channels [5-8]. Unfortunately, however, it is known that the 
obtained hydrophilicity is only temporary, namely, hydrophobicity recovery occurs within a few days by a combination of diffusive burial of polar groups in the bulk and condensation of silanol groups formed by plasma treatment and consequent cross-linking [9]. Hence, silanization [10], adsorbed coatings [11], and polymer grafting [12-15] are reported to be effective for forming a thin and stable coating on PDMS to achieve sustained hydrophilicity.

Recently a polymer family containing phosphorylcholine with a methacrylate group 2-methacryloyloxyethyl phosphorylcholine (MPC) polymer has attracted considerable attention in biointerface engineering [16]. Since the MPC copolymers have structures similar to the cell membrane, they show excellent biocompatibility because of reduced nonspecific protein adsorption on the surface. More recently, Ishihara's group reported the successful application of some MPC copolymers to the surface tailoring of silica microfluidic devices [17].

In this study, we investigated the coating of biomimetic polymer on PDMS surfaces by the combinative use of photochemical oxidation upon vacuum ultraviolet (VUV) irradiation to form surface silanol and subsequent dip-coating of phospholipid copolymer containing MPC and 3-methacryloxyethyl triethoxysilane (METESi). Specifically, changes in the PDMS surface after VUV irradiation and their effects on the subsequent MPC copolymer coating were comprehensively examined with various irradiation times. Excellent reduction of nonspecific protein adsorption on the surface and also the formation of a micropatterned MPC copolymer coating using a photomask were demonstrated.

\section{Experimental}

\subsection{Materials}

The PDMS precursor (Silpot 184®) and PDMS cross-linker (catalyst of Silpot 184®) were purchased from Toray-Dow Corning Co., Japan. MPC was synthesized by a previously described method [16] and recrystallized from acetonitrile. Poly(MPC-co-METESi) was synthesized by the same method using the monomers of MPC and METESi. The structure of the copolymer is shown in Fig. 1. Here, the METESi unit acts as a silane coupling agent and can be chemically bonded with silanol groups formed on the oxidized PDMS. Bovine serum albumin conjugated with fluorescein isothiocyanate (BSA-FITC) was purchased from Molecular Probes, Inc., USA, and used for the evaluation of the nonspecific adsorption of protein on the surface.

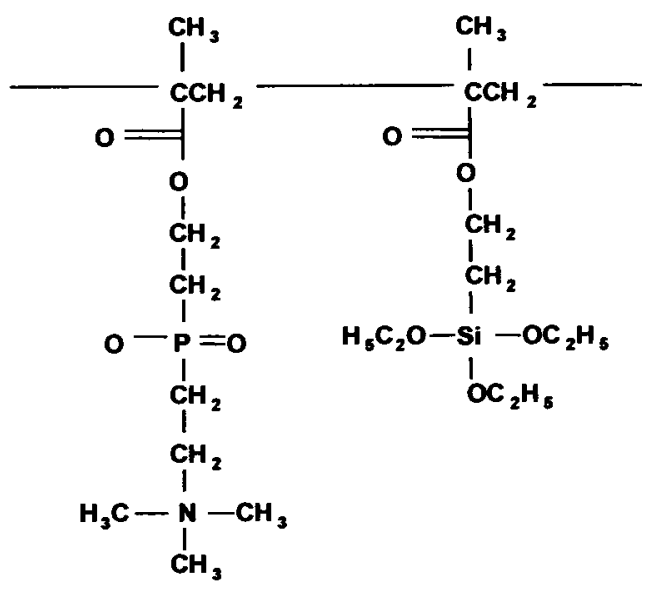

Fig. 1. Structure of MPC-co-METESi.

\subsection{Preparation of thin PDMS films}

The PDMS precursor and cross-linker were mixed at the weight ratio of 10:1. Mixtures of 10 $\mathrm{mL}$ volume were coated on flat glass substrates with the dimensions of 30 by 30 by $0.5 \mathrm{~mm}$ using a spin-coater at $5000 \mathrm{rpm}$ for $60 \mathrm{~s}$ and subsequently polymerized at $100^{\circ} \mathrm{C}$ for $2 \mathrm{~min}$ to attain cross-linked PDMS films.

\subsection{VUV irradiation and subsequent MPC copolymer coating on PDMS}

Figure 2 shows a schematic of the VUV treatment apparatus employed in this study. Exposure to VUV light at a wavelength of $172 \mathrm{~nm}$ was performed with a VUV Xe excimer lamp (lamp type: $172 / 120 \mathrm{Z}(\mathrm{HV})$, Heraeus Noblelight $\mathrm{GmbH}$, Germany) of $4 \mathrm{~cm}$ diameter and $12 \mathrm{~cm}$ length, mounted in a stainless-steel vacuum chamber.

The prepared PDMS thin films were set in the VUV curing chamber. After evacuation to $10 \mathrm{~Pa}$ using a rotary pump, VUV irradiation was carried out in a $100 \mathrm{~Pa}$ oxygen atmosphere at $36 \mathrm{~mW} / \mathrm{cm}^{2}$ for $30 \mathrm{~s}$ so that silanol groups were formed on 
PDMS surfaces. Subsequently, poly(MPC-coMETESi) was coated onto VUV-treated PDMS surfaces as follows. The PDMS films were dipped into MPC copolymer solution of $0.5 \mathrm{wt} \%$ in ethanol for approximately $10 \mathrm{~s}$ and placed in a same-solvent-vapor atmosphere at room temperature for $20 \mathrm{~min}$. Finally they were dried in air at $70^{\circ} \mathrm{C}$ for $4 \mathrm{~h}$.

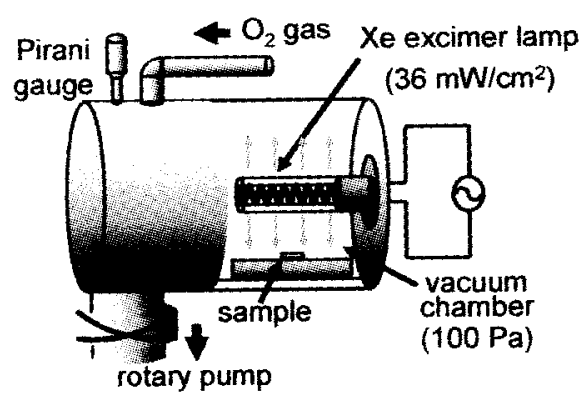

Fig. 2. Schematic diagram of UV treatment apparatus employed in this study.

\subsection{Surface characterization}

Contact Angle Measurements: Water contact angles were measured on PDMS films VUV-cured with various irradiation times. A droplet of deionized water was placed on the air-side surface of a film at room temperature. Our original contact angle measurement system, which consists of a humidity chamber and microscope with a long focal length, was used.

Infrared Spectroscopy: Attenuated total-reflection Fourier transform infrared (ATR-FTIR) spectra were measured from modified and unmodified PDMS surfaces using an IR spectrometer (FTIR-700, JASCO Co., Tokyo Japan) under dry conditions. The spectra were recorded from 650 to $4600 \mathrm{~cm}^{-1}$ at a $4 \mathrm{~cm}^{-1}$ resolution. A single-beam reference spectrum of a freshly cleaned germanium crystal prism with an incident angle of $45^{\circ}$ was recorded before the measurements and used as the background spectrum.

2.5 Evaluation of nonspecific adsorption of protein on the surface

Protein adsorption evaluation was conducted as follows. PDMS samples coated with MPC copolymer were immersed in phosphate-buffered saline (PBS; $\mathrm{pH}=7.4$. ionic strength $=1.0 \mathrm{~mol} / \mathrm{L}$ ) for $2 \mathrm{~h}$ prior to each experiment. Then, samples were dipped in BSA-FITC solution $(1.0 \mathrm{mg} / \mathrm{mL}$ in PBS buffer) for $20 \mathrm{~min}$ at $37^{\circ} \mathrm{C}$, and then rinsed by gently shaking 50 times in fresh PBS. Finally, the adsorbed protein was visually observed using a sensitive fluorescence microscope (ECLIPSE TE 2000-U, Nikon, Japan) equipped with both a chilled CCD camera (C5985, Hamamatsu Photonics K.K., Japan) and an image intensifier (C8600, Hamamatsu Photonics K.K., Japan). The fluorescence intensity quantified from recorded images using an image processing and analysis program, Scion Image (Scion Corp., USA), was adopted as the measure of the amount of adsorbed BSA-FITC.

2.6 MPC copolymer coating on PDMS with locally oxidized surface

One important feature of the present surface modification process is the possibility of simple patterning using a photomask. To demonstrate this advantage, a metal stencil mask with a $100 \mu \mathrm{m}$ mesh was set on PDMS samples during VUV irradiation. VUV was irradiated for $30 \mathrm{~s}$ and then MPC copolymer was coated in the same way as described in section 2.3.

\section{Results and Discussion}

\subsection{Surface characterization}

Firstly, the water contact angle was measured to evaluate the surface hydrophilicity of PDMS VUV-cured with various irradiation times. As shown in Fig. 3, the contact angle dropped rapidly within $20 \mathrm{~s}$ and, reached 0 degree at $50 \mathrm{~s}$, while further irradiation caused a gradual increase. According to the literature, the initial drop is mainly ascribed to the photochemical conversion of surface methylsilane groups to silanol groups [18]. Further VUV irradiation results in the subsequent degradation of the polymer and the formation of silicon oxide. The slight recovery of hydrophobicity observed after $100 \mathrm{~s}$ is believed to be caused by the increase in the nanoscopic surface roughness on the silicon oxide thin layer.

Secondly, in order to confirm the change in chemical bonds on PDMS surfaces after VUV irradiation, IR spectroscopy was conducted. Figure 4 
shows the ATR-FTIR spectra obtained from the PDMS samples soon after VUV irradiation. With the increase of VUV irradiation time, the broad absorption band centered at $3300 \mathrm{~cm}^{-1}$, attributed to silanol groups, gradually increased, while absorption peaks corresponding to the methyl group decreased, as was observed at 2962,1259 , and 796 $\mathrm{cm}^{-1}$. Such changes are in accordance with the successive substitution of methyl groups by hydroxyl groups, as reported previously [18].

Moreover, IR spectra were similarly measured from the samples after coating the MPC copolymer. With the increase of the irradiation time, we observed the gradual increase of the absorption peak at $1620 \mathrm{~cm}^{-1}$, which is assigned to the carbonyl groups that exist in MPC copolymer.

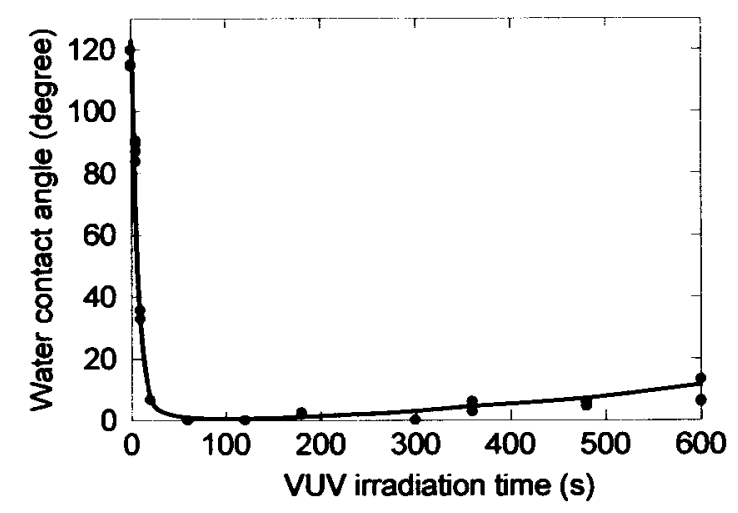

Fig. 3. Water contact angle versus VUV irradiation time.

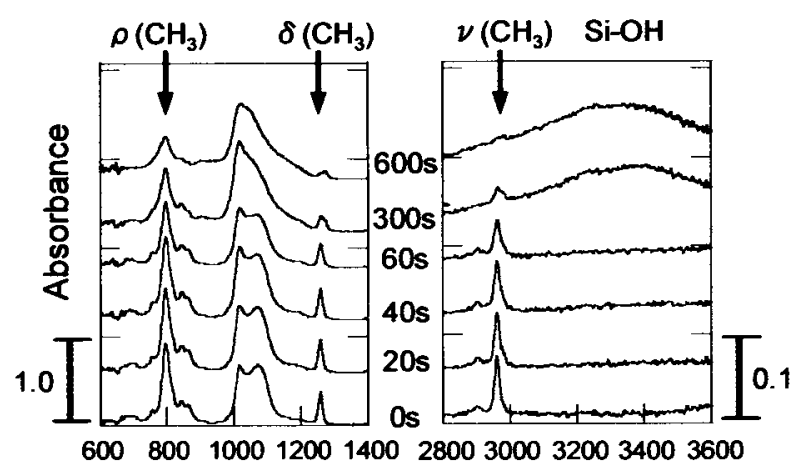

Wavenumber $\left(\mathrm{cm}^{-1}\right)$

Fig. 4. FT-IR spectra from PDMS surfaces exposed to VUV light in oxygen ambient for different irradiation times.
3.2. Evaluation of nonspecific protein adsorption

Figure 5 shows the change in the intensity of fluorescence from BSA-FITC adsorbed on MPC copolymer-coated PDMS surfaces as a function of VUV irradiation time. At $0 \mathrm{~s}$, namely, without VUV irradiation, MPC copolymer cannot bind on the PDMS surfaces, thus nonspecific adsorption of BSA was strongly observed. On the other hand, at the optimum VUV irradiation time of around $30 \mathrm{~s}$, significant reduction of BSA adsorption by as much as $90 \%$ was successfully achieved owing to the high-density MPC copolymer coating. In addition, it is noted that a gradual increase in BSA adsorption was observed with excessively long VUV irradiation time.

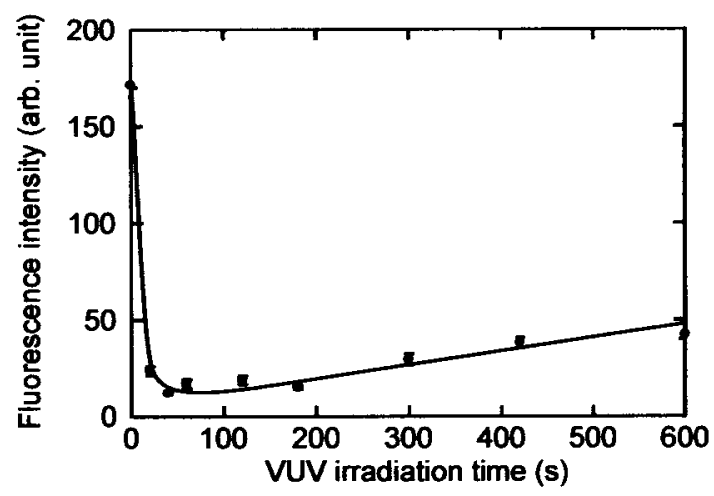

Fig. 5. Fluorescence intensity from BSA-FITC adsorbed on PDMS samples coated with MPC copolymer versus VUV irradiation time.

3.3. Micropatterned oxidation and MPC copolymer coating on PDMS

The coating of patterned MPC copolymer on the PDMS surface was performed by photochemical oxidation with a stencil mask and subsequent coating of poly(MPC-co-METESi) via the silylation reaction at the silanol groups on the selectively oxidized PDMS surface. Figure 6 shows the fluorescence-microscope image of BSA-FITC adsorbed on the PDMS sample with a patterned MPC coating. Pattern dimensions were in good accordance with those of the stencil mask. The clear contrast between bright and dark areas demonstrates that nonspecific adsorption of BSA-FITC was effectively suppressed only in the regions where MPC copolymer was selectively coated. 


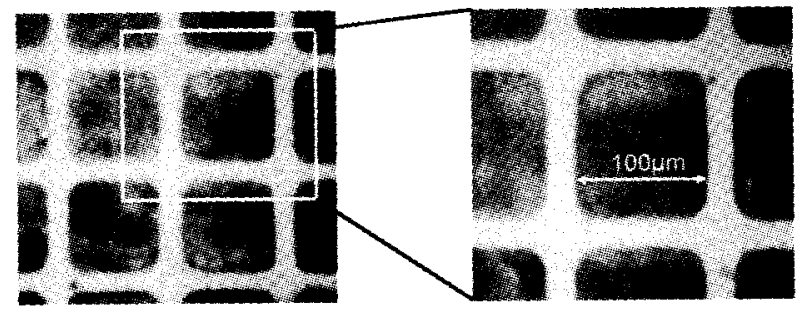

Fig. 6. Patterned adsorption of BSA-FITC was clearly observed on a PDMS sheet with a micropatterned MPC copolymer coating on its surface. Nonspecific adsorption occurred at the area where VUV light was not irradiated and consequently, MPC copolymer was not bound there.

\section{Conclusion}

In this paper, we reported a simple but reliable surface modification process for coating a biomimetic polymer to PDMS, which is widely used as a biodevice material. Photochemical oxidation by VUV irradiation followed by the coating of poly(MPC-co-METESi) to PDMS surfaces via the silylation reaction enabled us to form a dense MPC copolymer coating that can reduce the nonspecific adsorption of BSA by as much as $\mathbf{9 0 \%}$ compared with the nontreated PDMS surface. Moreover, this process is readily adaptable to the micropatterned surface coating using a photomask during VUV irradiation. These features are effective for selective protein adsorption and cell adhesion at desired locations on biodevices.

\section{References}

1. P. Lenz, C. M. Ajo-Franklin, and S. G. Boxer, Langmuir, 20 (2004) 11092.

2. J. C. McDonald and G. M. Whitesides, Acc. Chem. Res., 35 (2002) 491.

3. W. Hellmich, J. Regtmeier, T. T. Duong, R. Ros,
D. Anselmetti, and A. Ros, Langmuir, 21 (2005) 7551.

4. S. K. Sia and G. M. Whitesides, Electrophoresis, 24 (2003) 3563.

5. D. C. Duffy, J. C. McDonald, O. J. A. Schueller, and G. M. Whitesides, Anal. Chem., 70 (1998) 4974.

6. S. Hu, X. Ren, M. Bachman, C. E. Sims, G. P. Li, and N. Allbritton, Anal. Chem., 74 (2002) 4117.

7. M. J. Owen, and P. J. Smith, J. Adhesion Sci. Technol., 8 (1994) 1063.

8. L. Chen, J. Ren, R. Bi, and D. Chen, Electrophoresis, 25 (2004) 914.

9. M. Morra, E. Occhiello, R. Marola, F. Garbassi, P. Humphrey, and D. Johnson, J. Colloid Interface Sci., 137 (1990) 11.

10. B. A. Grzybowski, R. Haag, N. Bowden, and G. M. Whitesides, Anal. Chem., 70 (1998) 4645.

11. Y. Liu, J. C. Fanguy, J. M. Bledsoe, and C. S. Henry, Anal. Chem., 72 (2000) 5939.

12. S. Hu, X. Ren, M. Bachman, C. E. Sims, G. P. Li, and N. L. Allbritton, Anal. Chem., 74 (2002) 4117.

13. S. Hu, X. Ren, M. Bachman, C. E. Sims, G. P. Li, and N. L. Allbritton, Anal. Chem., 76 (2004) 1865.

14. G. Sui, J. Wang, C. C. Lee, W. Lu, S. P. Lee, J. V. Leyton, A. M. Wu, and H. R. Tseng, Anal. Chem., 78 (2006) 5543.

15. D. Wu, B. Zhao, Z. Dai, J. Qin, and B. Lin, $L a b$ Chip, 6 (2006) 942.

16. K. Ishihara, T. Ueda, and N. Nakabayashi, Polym. J., 22 (1990) 355.

17. Y. Xu, M. Takai, T. Konno, and K. Ishihara, Lab Chip, 7 (2007) 199.

18. V-M. Graubner, R. Jordan, O. Nuyken, B. Schnyder, T. Lippert, R. Koetz, and A. Wokaun, Macromolecules, 37 (2004) 5963. 